\title{
CDP7657, an anti-CD40L antibody lacking an Fc domain, inhibits CD40L-dependent immune responses without thrombotic complications: an in vivo study
}

Anthony Shock ${ }^{1 *}$, Linda Burkly², lan Wakefield ${ }^{1}$, Christopher Peters ${ }^{3}$, Ellen Garber ${ }^{2}$, Janine Ferrant ${ }^{2}$,

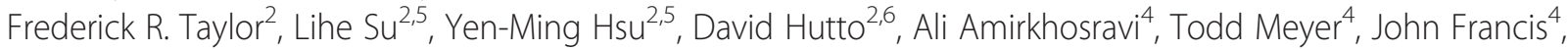
Sarah Malcolm", Martyn Robinson ${ }^{1}$, Derek Brown ${ }^{1,7}$, Stevan Shaw ${ }^{1}$, Roland Foulkes ${ }^{1,8}$, Alastair Lawson ${ }^{1}$, Olivier Harari ${ }^{1}$, Timothy Bourne ${ }^{1}$, Alison Maloney ${ }^{1}$ and Neil Weir ${ }^{1}$

\begin{abstract}
Introduction: CD40 ligand (CD40L) blockade has demonstrated efficacy in experimental autoimmune models. However, clinical trials of hu5C8, an anti-human CD40L IgG antibody, in systemic lupus erythematosus (SLE) were halted due to an increased incidence of thrombotic events. This study evaluated CDP7657, a high affinity PEGylated monovalent Fab' anti-CD40L antibody fragment, to assess whether an Fc-deficient molecule retains efficacy while avoiding the increased risk of thrombotic events observed with hu5c8.

Methods: The potency and cross-reactivity of CDP7657 was assessed in in vitro assays employing human and non-human primate leukocytes, and the capacity of different antibody formats to activate platelets in vitro was assessed using aggregometry and dense granule release assays. Given the important role CD40L plays in regulating humoral immunity, in vivo efficacy was assessed by investigating the capacity of Cynomolgus monkeys to generate immune responses to the tetanus toxoid antigen while the potential to induce thrombotic events in vivo was evaluated after repeat dosing of antibodies to Rhesus monkeys. A PEGylated anti-mouse CD40L was generated to assess efficacy in the New Zealand Black/White (NZB/W) mouse model of SLE.
\end{abstract}

Results: CDP7657 dose-dependently inhibited antigen-specific immune responses to tetanus toxoid in Cynomolgus monkeys, and in contrast to hu5c8, there was no evidence of pulmonary thrombovasculopathy in Rhesus monkeys. Aglycosyl hu5c8, which lacks Fc receptor binding function, also failed to induce thrombotic events in Rhesus monkeys. In vitro experiments confirmed that antibody constructs lacking an Fc, including CDP7657, did not induce human or monkey platelet activation. A PEGylated monovalent Fab' anti-mouse CD40L antibody also inhibited disease activity in the NZB/W mouse model of SLE after administration using a therapeutic dosing regimen where mice received antibodies only after they had displayed severe proteinuria.

Conclusions: These findings demonstrate for the first time that anti-CD40L antibodies lacking a functional FC region do not induce thrombotic events in Rhesus monkeys and fail to activate platelets in vitro but, nevertheless retain pharmacological activity and support the investigation of CDP7657 as a potential therapy for systemic lupus erythematosus and other autoimmune diseases.

\footnotetext{
*Correspondence: Tony.Shock@ucb.com; linda.burkly@biogenidec.com

'UCB Pharma, 216 Bath Road, Slough, Berkshire SL1 4EN, UK

${ }^{2}$ Biogen Idec, Inc., 12 Cambridge Center, Cambridge, MA 02142, USA

Full list of author information is available at the end of the article
} 


\section{Introduction}

CD40 ligand (CD40L), or CD154, is expressed on activated $\mathrm{T}$ lymphocytes, and through interactions with its receptor CD40, plays a pivotal role in regulating the interplay between $\mathrm{T}$ cells and other cell types [1-3]. The CD40L/CD40 pair is known to mediate cognate $\mathrm{T}$ cell help for B cells, resulting in increased B cell proliferation and differentiation, antibody production and isotype class switching. CD40L also promotes the formation of germinal centers in lymph nodes and B-cell survival. CD40L therefore holds significant promise as a therapeutic target in autoimmune disease, and blockade of CD40L has been shown to be highly efficacious in several inflammatory and autoimmune model systems [4-8].

CD40L has also been detected on other immune and non-lymphoid cells [3] and is present in platelets $[9,10]$. Within seconds of being stimulated by platelet agonists, CD40L is presented on the surface of platelets, and is subsequently shed as soluble CD40L (sCD40L) [9]. Furthermore, a role for platelet-derived CD40L in regulating adaptive immunity and thrombosis has been suggested [11-14].

$\mathrm{Hu} 5 \mathrm{c} 8$, a monoclonal $\mathrm{IgG}_{1}$ antibody against $\mathrm{CD} 40 \mathrm{~L}$, was evaluated in clinical trials for a range of autoimmune diseases. Results from a phase 2 study in patients with systemic lupus erythematosus (SLE) were encouraging, with significant reductions in disease biomarkers, including circulating levels of autoantibodies and marked increases in C3 levels [15-17]. However, despite this promising evidence of clinical effect, further development of hu5c8 was discontinued because of an increased incidence of treatment-emergent cardiovascular thrombotic events (TEs) [18]. More recently, in a study of hu $5 \mathrm{c} 8$ in the Rhesus monkey, numerous TEs including pulmonary vascular thrombi and vasculopathy were found after administration of hu5c8 [19], suggesting that the Rhesus monkey is a relevant and sensitive pre-clinical model for induction of TEs by anti-CD40L $\mathrm{IgG}_{1}$ antibodies in humans. The mechanism by which hu5c8 induces TEs in humans remains unclear. In vitro analyses have shown that immune complexes (IC) consisting of sCD40L and an anti-CD40L monoclonal antibody can trigger platelet aggregation [12, 20]. This effect seems to be dependent on the anti-CD40L monoclonal antibody carrying a functional Fc region, and signaling through the FcyRIIa (CD32a) Fc receptor on the platelet surface $[12,20,21]$.

To evaluate whether one could achieve the therapeutic potential of CD40L blockade while removing the TE risk hypothesized to be due to platelet activation, this paper describes the generation and testing of an anti-CD40L antibody fragment lacking a functional Fc region. To increase the circulating half-life of this monovalent Fab' antibody fragment, a $40 \mathrm{kDa}$ polyethylene glycol (PEG) moiety was conjugated via a chemical linker, and this new molecule was designated CDP7657. CDP7657 is currently undergoing phase 1 clinical trials in patients with SLE.

A series of experimental studies was conducted utilizing a variety of antibody constructs, including CDP7657 and aglycosyl IgG forms of anti-CD40L antibodies, in order to determine the importance of Fc function and valency for the efficacy and safety of anti-CD40L antibodies. Here, we describe findings demonstrating that CDP7657 inhibits antigen-specific immune responses to the tetanus toxoid (TT) antigen in Cynomolgus monkeys. The profound efficacy of an anti-mouse CD40L monovalent PEGylated Fab' fragment was also demonstrated in a murine model of SLE. In addition, the Fc region of anti-CD40L antibodies was shown to be important for the activation of platelets in vitro and for the induction of TEs in Rhesus monkeys in vivo.

\section{Methods}

Antibodies and reagents

Anti-CD40L antibodies used were: hu5c8, a humanized anti-human $\mathrm{CD} 40 \mathrm{~L}$ intact $\mathrm{IgG}_{1}$ monoclonal antibody (Biogen Idec, Cambridge, MA, USA); aglycosyl hu5c8, a mutant form of hu5c8 with reduced FcR binding (Biogen Idec, MA, USA); CDP7657, a monovalent Fab' PEGylated anti-CD40L antibody (UCB Pharma, Slough, UK); M90, a mouse anti-human $\mathrm{CD} 40 \mathrm{~L}$ intact $\mathrm{IgG}_{1}$ monoclonal antibody (hybridoma from ATCC, Manassas, VA, USA); MR1 Fab' PEG, a monovalent, murinized Fab' PEGylated antimouse CD40L (UCB Pharma, Slough, UK). Soluble CD40L was from Peprotech (Rocky Hill, NJ, USA), phycoerythrin (PE)-labeled CD40-Fc fusion protein was from Ancell (Bayport, MN, USA) and thrombin receptor agonist peptide (TRAP) was from Sigma (St Louis, MO, USA).

\section{Activity and cross-reactivity experiments}

BIAcore (surface plasmon resonance) experiments were performed to calculate the affinity $\left(K_{\mathrm{D}}\right.$, equilibrium dissociation constant) of CDP7657 for CD40L and involved capturing the antibody using an anti-human Fab' antibody and then titrating human $\mathrm{SCD} 40 \mathrm{~L}$ protein over the immobilized antibodies. The $K_{D}$ for hu5c8 was assessed after capture with an anti-Fc antibody. Potency was assessed in two flow cytometry-based, cell-based assays: the binding of a PE-labeled CD40-Fc fusion protein to CD40Lexpressing D1.1 Jurkat cells and an assay of T-celldependent $\mathrm{B}$ cell activation, involving overnight co-culture of CD40L-expressing D1.1 cells with the Ramos B cell line and monitoring intercellular adhesion molecule 1 (ICAM-1) expression on the latter cells. Non-human primate cross-reactivity of CDP7657 was measured in saturation binding experiments using a broad-range antibody titration on peripheral blood lymphocytes isolated from 
humans, Rhesus monkeys and Cynomolgus monkeys and activated in vitro to express CD40L. Human blood samples were obtained under a licence (\#12504) granted to UCB under Section 16 (2) (e) (ii) of the Human Tissue Act (UK) and all donors provided their written consent to participate.

\section{Immune response to TT in a Cynomolgus monkey model} Groups of six Cynomolgus monkeys $(n=3 /$ sex) were administered a single intravenous (i.v.) dose of antibody or saline and, after $4 \mathrm{~h}$ or $48 \mathrm{~h}$, an intramuscular (i.m.) dose of TT $(0.5 \mathrm{~mL})$, except in the dose-response study, where $20 \mathrm{mg} / \mathrm{kg}$ IgG antibodies and $40 \mathrm{mg} / \mathrm{kg}$ PEGylated antibodies were used. Plasma was isolated at various time intervals for anti-TT analyses by ELISA. Plates were coated with TT, incubated with plasma samples and after washing, binding was detected using horseradish peroxidase (HRP)-conjugated anti-monkey IgG antibodies. Mean anti-TT IgG titer \pm standard deviation were calculated and where appropriate, one-way analysis of variance (ANOVA) was performed. As parametric statistical methods were used, the data should be declared to have been found normally distributed and of equal variance.

\section{NZB/W SLE nephritis mouse model}

All experiments were conducted under protocols approved by the Animal Welfare and Ethical Review Body of UCB (UK) in accordance with the Animals (Scientific Procedures) Act 1986. Female NZB/NZW $F_{1}$ mice (Harlan, UK) aged 18 weeks, were housed in cages of seven or eight mice, in a temperature- and humiditycontrolled room, with 12-h light-dark cycles. All animals received food and water ad libitum.

Female NZB/NZW $F_{1}$ mice were assessed for disease progression from 18 weeks of age with levels of protein $(\mathrm{mg} / \mathrm{dL})$ in the urine measured by Albustix (Bayer, Leverkusen, Germany) twice a week. After recording a proteinuria level $\geq 300 \mathrm{mg} / \mathrm{dL}$ on one occasion, mice were randomized into one of two dosing groups and dosed subcutaneously (s.c.) with either saline or $100 \mathrm{mg} / \mathrm{kg}$ MR1 Fab' PEG. Animals entered disease remission when a proteinuria level $\leq 100 \mathrm{mg} / \mathrm{dL}$ was measured on two consecutive occasions, and disease relapse was classified as animals that scored $\geq 300 \mathrm{mg} / \mathrm{dL}$ on two consecutive occasions. Remission and relapse data were subjected to the Mann-Whitney $U$ test to compare MR1 Fab' PEG to saline control. Survival data were analyzed by the log-rank test.

\section{Platelet activation by anti-CD40L antibodies Platelet aggregometry}

The protocol for obtaining human blood for all platelet studies was approved by the Florida Hospital Institutional Review Board and donors provided their written consent to participate. Washed platelet aggregation studies were performed as described previously [22]. Briefly, washed platelets (four human donors, two Rhesus monkeys) were suspended in HEPES buffer, and platelets were adjusted to $250 / \mathrm{nL}$ and analyzed by light transmission aggregometry (300 $\mu \mathrm{L} /$ assay). Baseline traces were established and preformed anti-CD40L ICs were added to a final concentration of $200 \mathrm{nM}$. For studies with preformed IC, antibody formats (hu5c8, aglycosyl hu5c8 or CDP7657) were combined with SCD40L in a ratio of one antibody to one antigen homotrimer, the molar stoichiometric equivalent of this being one antibody per three sCD40L antigen monomers. Aggregations were monitored for at least 6 minutes.

\section{Measurement of platelet dense granule release}

The serotonin release assay was performed as described previously [21]. Briefly, platelets in platelet-rich plasma (one human donor, four Rhesus monkeys) were labeled with ${ }^{14} \mathrm{C}$-radiolabeled serotonin $(0.1 \mu \mathrm{Ci} / \mathrm{mL})$, incubated for 45 minutes at $37{ }^{\circ} \mathrm{C}$, washed in apyrase buffer (to prevent spurious activation), then re-suspended in albumin-free Tyrode's assay buffer. Antibody solutions were clarified by centrifugation (16,100 rpm, 15 minutes) before adding to assay wells to a final concentration of $500 \mathrm{nM}$. For studies with preformed IC, antibody formats (hu5c8, aglycosyl hu5c8 or CDP7657) were combined with SCD40L in a ratio of one antibody to one antigen homotrimer, the molar stoichiometric equivalent of this being one antibody per three sCD40L antigen monomers. ICs, pre-incubated for 5-20 minutes prior to assay, were then added to microtiter plate wells to achieve a final concentration of $500 \mathrm{nM}$.

\section{Assessment of TE in Rhesus monkeys}

Studies in Cynomolgus and Rhesus monkeys were conducted at Charles River Laboratories (NV, USA), followed international Good Laboratory Practice (GLP) standards and were conducted in accordance with the regulations of the USDA Animal Welfare Act (2010) and in compliance with the testing facility's Animal Welfare Assurance (A4112-01) filed with the National Institutes of Health $(\mathrm{NIH})$; the protocols were approved by the Charles River Laboratories Institutional Animal Care \& Use Committee.

A head-to-head comparative 8-week study investigated the occurrence of TEs with the monovalent Fab' PEG CDP7657 and two intact monoclonal antibodies, hu5c8 and aglycosyl hu5c8. These agents were administered i.v. at $50 \mathrm{mg} / \mathrm{kg} /$ week for 8 weeks to Rhesus monkeys ( $\mathrm{n}=$ $4 /$ sex/group) and compared with saline-injected controls $(\mathrm{n}=2 / \mathrm{sex})$.

A 3-month GLP pivotal safety study assessed potential dose-dependent TE occurrence with CDP7567 and general 
potential for toxicity. CDP7567 was administered at 20, 50 or $200 \mathrm{mg} / \mathrm{kg} /$ week i.v. ( $\mathrm{n}=5 / \mathrm{sex} /$ group) for 12 weeks. Control animals $(n=10 / \mathrm{sex})$ received saline. At the end of the treatment period, additional satellite groups $(n=1-4$ / sex/group) were used to assess any reversibility effects over 4 and 6 months. For both studies, monoclonal antibodies were administered by bolus i.v. injection over approximately 60 s. Standard toxicology endpoints were monitored. At necropsy, lungs were insufflated with neutralbuffered formalin and allowed to fully fix by immersion in additional neutral-buffered formalin.

Macroscopic examination of pulmonary tissues included partial dissection of pulmonary and bronchial arteries. A total of 29 histopathology sections were taken from each animal. Five histopathology sections were collected from each of the four largest lung lobes (left and right diaphragmatic, and left and right apical). Three sections were prepared from the three smallest lung lobes (left and right middle lobes, and intermediate lobe).

\section{Results}

CDP7657 inhibits the humoral immune response

CDP7657 has a very high affinity for CD40L and potently neutralizes CD40L in functional cell-based assays, measuring CD40 binding to CD40L, and CD40L-dependent B cell activation (Table 1). It had similar affinity for human and non-human primate (Rhesus monkey and Cynomolgus monkey) CD40L (Table 2), which was expected as the CD40L protein sequences of Rhesus monkeys and Cynomolgus monkeys are identical, and share $98 \%$ of their identity with human CD40L. CDP7657 possesses a higher affinity than hu5c8 and both antibodies have similar activities in cell-based assays (Table 1). CDP7657 does not bind to rodent $\mathrm{CD} 40 \mathrm{~L}$ and, therefore, it was necessary to investigate the in vivo efficacy of CDP7657 in non-human primates. The model employed monitored the impact of CDP7657 on the antibody response to TT in Cynomolgus monkeys, a model previously used to evaluate hu5c8 efficacy [5].

A single-dose study was conducted to assess the efficacy of CDP7657 at three dose levels (5, 20 and $60 \mathrm{mg} / \mathrm{kg}$ i.v.), compared with hu5c8 at $20 \mathrm{mg} / \mathrm{kg}$ i.v., a dose shown to be effective in previous studies with this antibody [23]. The pharmacokinetics were considered to be typical for the antibody constructs used, and were dose-proportional. Similar overall exposures were achieved with CDP7657
Table 2 CDP7657 cross-reactivity with human and non-human primate (Cynomolgus and Rhesus monkey) CD40L

\begin{tabular}{|c|c|c|c|c|c|c|}
\hline \multirow[t]{3}{*}{ Cell type } & \multicolumn{6}{|l|}{$K_{D}(n M)$} \\
\hline & \multicolumn{2}{|l|}{ Human } & \multicolumn{2}{|c|}{ Cynomolgus monkey } & \multicolumn{2}{|c|}{ Rhesus monkey } \\
\hline & Donor 1 & Donor 2 & Donor 1 & Donor 2 & Donor 1 & Donor 2 \\
\hline PBMC & 1.57 & 1.41 & 1.50 & 1.13 & 1.21 & 0.80 \\
\hline
\end{tabular}

PBMC peripheral blood mononuclear cell

and hu $5 \mathrm{c} 8$ at the same dose of $20 \mathrm{mg} / \mathrm{kg}$ i.v (Fig. 1 legend).

The serum IgG responses elicited by TT provocation were inhibited dose-dependently by CDP7657, and the inhibition was statistically significant with $60 \mathrm{mg} / \mathrm{kg}$ i.v. $(P<0.05)$ (Fig. 1). The degree of inhibition of the IgG response seen with CDP7657 at $60 \mathrm{mg} / \mathrm{kg}$ i.v. was similar to that achieved with hu5c8 at $20 \mathrm{mg} / \mathrm{kg}$ i.v. A similar pattern of inhibition was observed when the secondary immune response was assessed after administration of a second dose of antibody to the animals and re-challenge with TT (see Additional file 1). A reduction in potency was seen with an aglycosyl derivative of hu $5 \mathrm{c} 8$ compared to hu5c8 (Fig. 2). In this molecule, a single amino acid change in the Fc region prevents normal $\mathrm{N}$-glycosylation of the antibody [23]. This change does not alter the affinity of the antibody for CD40L, but does ablate antibody effector function including FcyR binding. Aglycosyl hu 5 c8 was shown to have a similar potency to CDP7657 in this model. Taken together, these data demonstrate that Fc function plays a role in the potency of antiCD40L antibodies in this in vivo model, although equivalent efficacy was achieved with CDP7657 when dosed at $60 \mathrm{mg} / \mathrm{kg}$.

\section{A murine anti-CD40L monovalent Fab' PEG antibody fragment inhibits disease in SLE-prone NZB/W F1 mice} To assess whether an anti-CD40L antibody construct lacking an Fc region retains efficacy in experimental models of autoimmune disease, a PEGylated monovalent Fab' antimouse antibody, MR1 Fab' PEG, was generated. Female NZB/NZW F1 mice spontaneously develop an autoimmune disease, which has many of the pathological features of human SLE. MR1 Fab' PEG demonstrates profound inhibition in this model when dosed prophylactically (i.e., before disease onset), but has also been assessed under more stringent conditions using a therapeutic dosing

Table 1 Affinity and potency of CDP7657 and hu5c8

\begin{tabular}{lll}
\hline Assay & CDP7657 & Hu5c8 \\
\hline BIAcore affinity $\left(K_{D}\right)$ & $7.9 \mathrm{pM}$ & $33 \mathrm{pM}$ \\
CD40 binding $\left(I C_{50}\right)$ & $44 \mathrm{ng} / \mathrm{mL}(490 \mathrm{pM})$ \\
CD40L-dependent B-cell activation (ICAM-1 expression) $\left(I C_{50}\right)$ & $83 \mathrm{ng} / \mathrm{mL}(920 \mathrm{pM})$ & $44 \mathrm{ng} / \mathrm{mL}(293 \mathrm{pM})$ \\
\hline
\end{tabular}

${ }^{*}$ Activity in this assay is very sensitive to the valency of anti-CD40L antibodies tested, and the $\mathrm{IC}_{50}$ for hu5c8 Fab is $977 \mathrm{ng} / \mathrm{mL}$ ( $\mathrm{n}=20$ ). CD40L CD40 ligand, ICAM intercellular adhesion molecule 1 


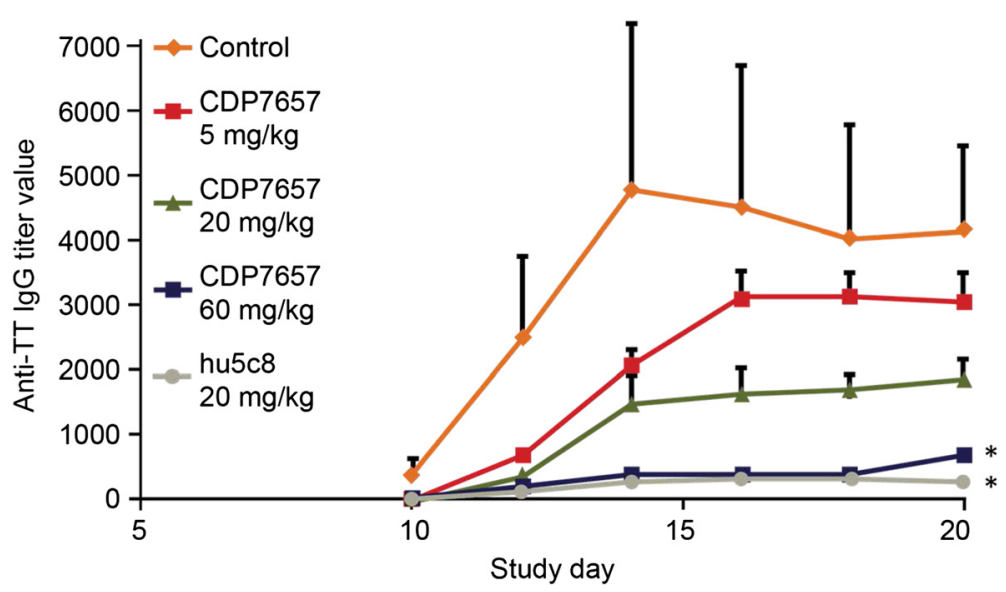

Fig. 1 Inhibition of humoral immune response in Cynomolgus monkeys: CDP7657 dose response. CDP7657 (various doses) was compared with a single dose of hu5c8 $(20 \mathrm{mg} / \mathrm{kg})$. Animals were administered a single dose of antibody or saline i.v. on day 1 and challenged with tetanus toxoid (TT) $4 \mathrm{~h}$ later. Data are expressed as the mean anti-TT IgG titer \pm standard deviation; ${ }^{*} P<0.05$ (one-way analysis of variance), compared with control, was considered to be statistically significant. Maximum concentration $\left(C_{\max }\right)$ and area under the concentration-time curve from time 0 until 19 days $\left(\mathrm{AUC}_{0-19}\right)$ values were calculated for each tested dose of antibody, day 19 being the last day anti- $T$ response was measured after anti-CD40 ligand administration. $C_{\max }$ was 163,704 and 2,088 $\mathrm{gg} / \mathrm{mL}$ for 5, 20 and $60 \mathrm{mg} / \mathrm{kg}$ doses of CDP7657, respectively. AUC $0-19$ was 713 , 2476 and 10,488 $\mu \mathrm{g} /$ day/mL for 5, 20 and $60 \mathrm{mg} / \mathrm{kg}$ CDP7657, respectively. $C_{\max }$ and $A \cup C_{0-19}$ for hu5c8 (20 mg/kg) were 414 and 3,186 $\mu \mathrm{g} /$ day $/ \mathrm{mL}$, respectively

regimen whereby mice were administered antibodies only after demonstrating high levels of proteinuria ( $>300 \mathrm{mg} / \mathrm{dL}$ ). Under these conditions, treatment with a single dose of MR1 Fab' PEG resulted in protection from disease, with $50 \%$ of animals entering remission (proteinuria level $\leq 100 \mathrm{mg} / \mathrm{dL}$ measured on two consecutive occasions) compared to a saline control group, resulting in increased survival of the treated animals (Fig. 3). These data show that a monovalent PEGylated anti-CD40L antibody fragment profoundly affects disease remission in an experimental model of SLE, as had previously been demonstrated for full-length anti-CD40L antibodies [24].

\section{Platelet activation by anti-CD40L antibodies requires} FcyRlla interaction

As well as confirming that CDP7657 was efficacious in both rodent and Cynomolgus monkey models, it was important to determine whether this construct mitigated the Fc-mediated platelet activation believed to be responsible for the prothrombotic events observed with hu5c8. Using platelet function tests, the possible prothrombotic activity of hu5c8, aglycosyl hu5c8 and CDP7657 was explored using human and Rhesus monkey blood.

In washed platelets from human healthy donors, immune complex (IC) consisting of hu5c8 with sCD40L

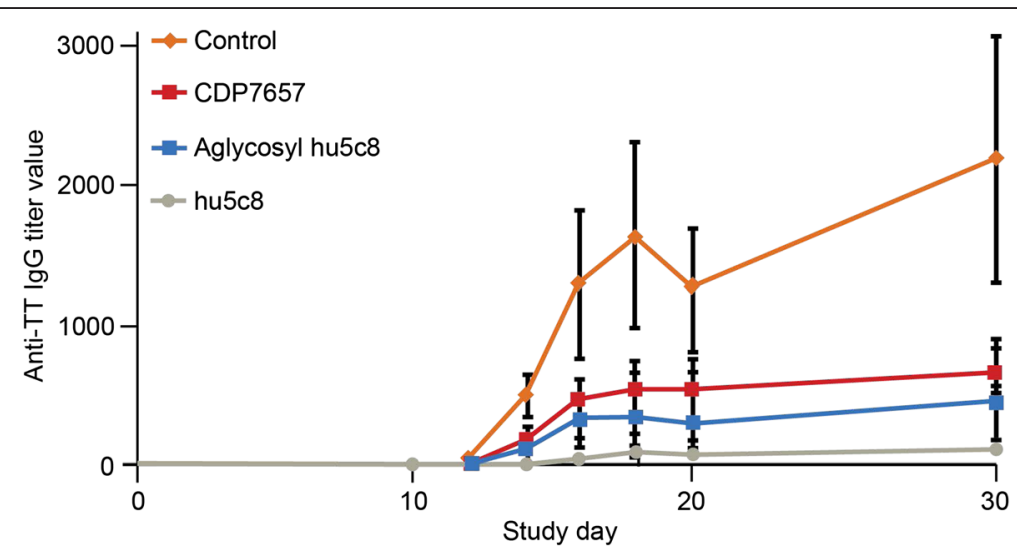

Fig. 2 Inhibition of humoral immune response in Cynomolgus monkeys: comparison of antibody formats. Animals received a single i.v. dose of antibody or saline 2 days prior to administration of tetanus toxoid (TT); the doses were $20 \mathrm{mg} / \mathrm{kg}$ for the lgG antibodies and $40 \mathrm{mg} / \mathrm{kg}$ for the PEGylated formats ( $n=6$ for all groups). The lgG anti-TT titers in plasma samples were assessed from days 10 to 31 

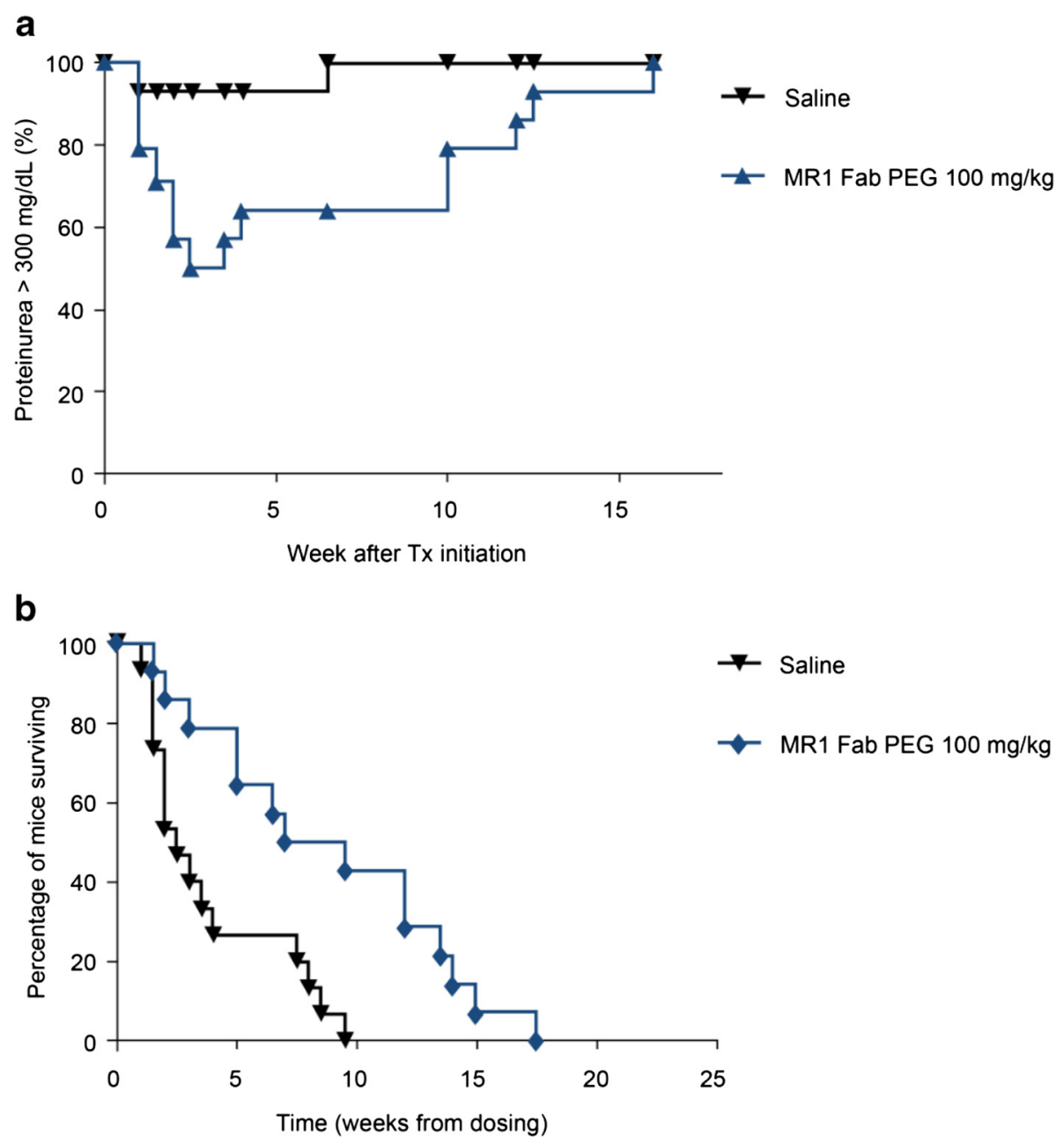

Fig. 3 Disease remission and increased survival after therapeutic dosing with MR1 Fab' polyethylene glycol (PEG) in NZB/NZW mice. a Remission and relapse of proteinuria levels in NZB/W mice after a single dose of $100 \mathrm{mg} / \mathrm{kg}$ MR1 Fab' PEG $(n=14)$ compared to saline control $(n=15)$. Data are expressed as the percentage of animals scoring $\geq 300 \mathrm{mg} / \mathrm{dL}$ proteinuria, with remission classified as two consecutive scores $\leq 100 \mathrm{mg} / \mathrm{dL}$ proteinuria; $P=0.0146$ (Mann-Whitney $U$ test) compared with saline control. $\mathbf{b}$ Percentage of mice surviving in each group over time; $P<0.0031$ (log-rank test) compared to saline control, and was considered to be statistically significant. Tx therapy

(hu5c8 IC) rapidly caused complete aggregation, whereas aglycosyl hu5c8 IC and CDP7657 IC were completely inactive (Fig. 4a). Hu5c8, aglycosyl hu5c8 and CDP7657 were all inactive in the absence of $\mathrm{sCD} 40 \mathrm{~L}$, as was sCD40L alone (see Additional file 2). M90, another antihuman CD40L antibody, also induced platelet aggregation in an intact murine IgG format, when combined with sCD40L. Additionally, hu5c8 IC consistently induced a strong serotonin dense granule release from isolated platelets (Fig. 4b) but CDP7657 and aglycosyl hu 5 c8 were completely inactive.

Comparable experiments performed using Rhesus monkey blood samples were consistent with the human findings. As shown in Fig. 4c, hu5c8 IC caused complete aggregation of washed Rhesus monkey platelets, whereas CDP7657 IC had no aggregatory effect. Similarly, hu5c8 IC induced a strong serotonin dense granule release from
Rhesus monkey platelets (Fig. 4d). Aglycosyl hu5c8 IC and CDP7657 IC, in addition to hu5c8, aglycosyl hu5c8, CDP7657 or sCD40L alone were completely inactive.

Overall, these data suggest that aglycosyl hu5c8 IC and CDP7657 IC do not induce platelet aggregation in both human and Rhesus monkey test systems; the same was true even when Rhesus monkey platelets were primed with sub-optimal ADP (See Additional file 3). These data are consistent with earlier reports showing that CD40L antibodies require $\mathrm{Fc}$-mediated signaling through FcyRIIa to activate platelets [12] and that aglycosyl hu5c8 (incapable of binding FcR) and antibody fragments lacking an Fc region are without activity in these assay systems.

Lack of evidence of TEs in Rhesus monkeys with CDP7657 Given that CDP7657 did not lead to activation of platelets from either humans or Rhesus monkeys, an evaluation to 


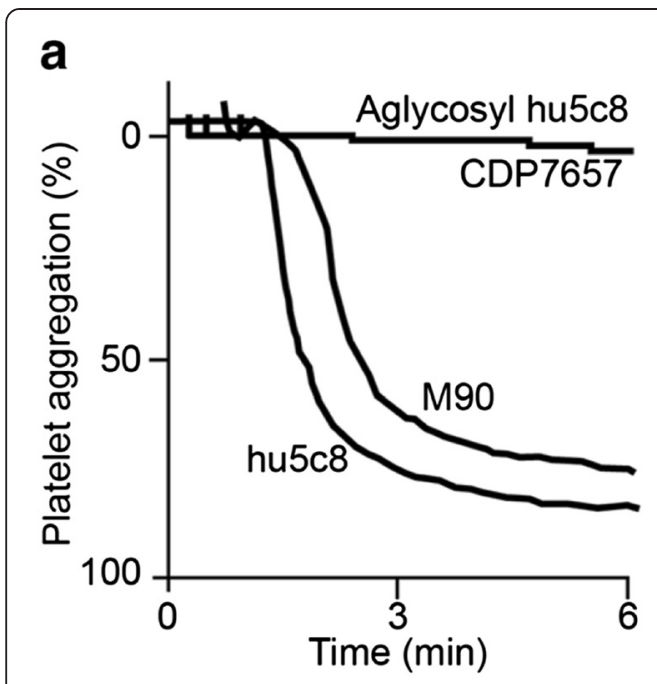

C

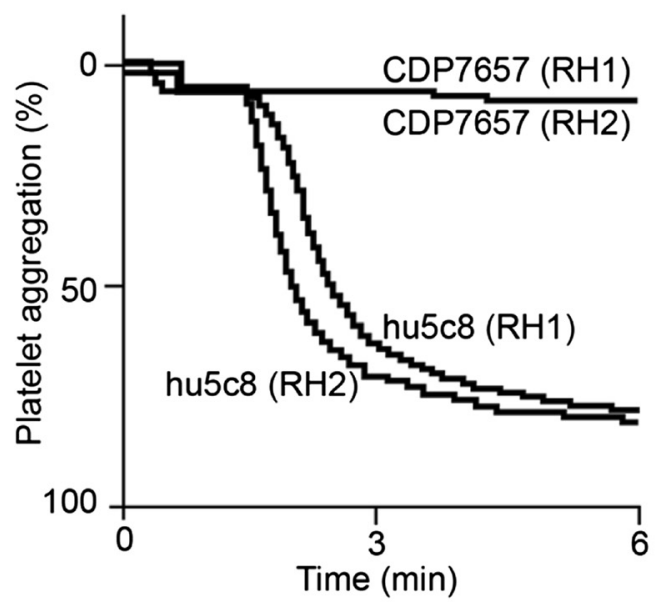

b

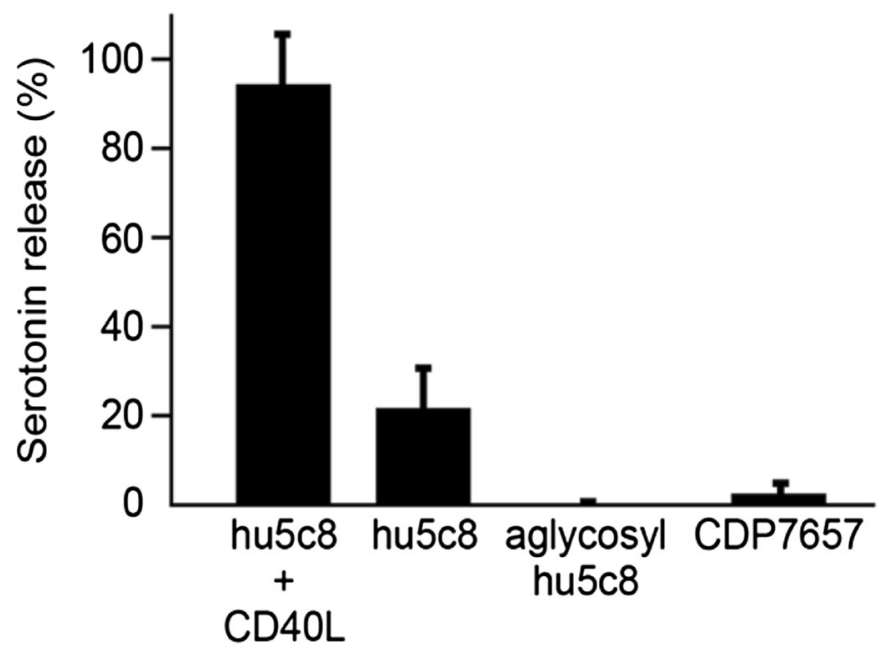

d

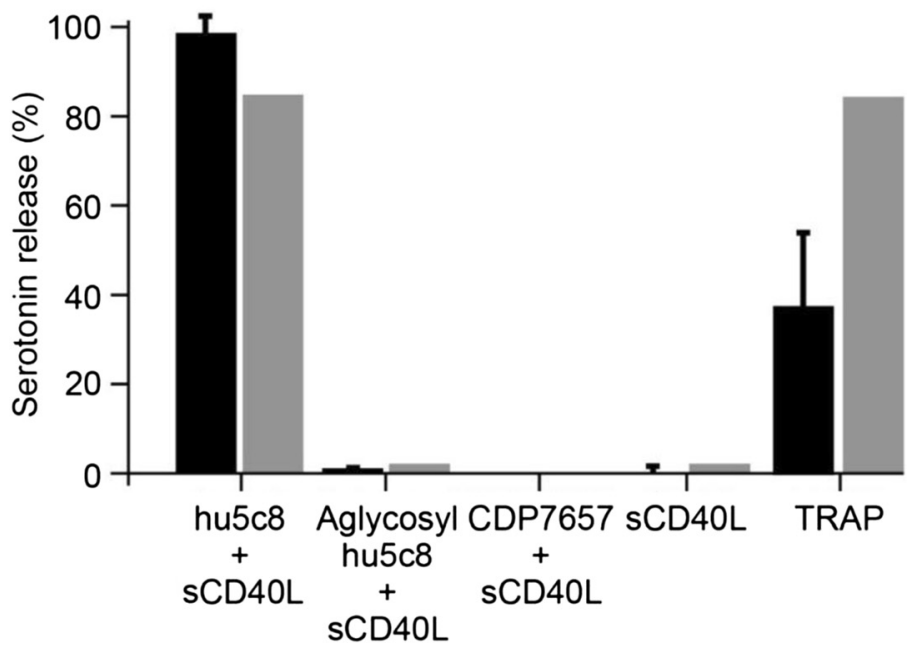

Fig. 4 Platelet activation by anti-CD40 ligand (anti-CD40L)antibodies requires FcyRlla interaction. a In vitro platelet aggregation isolated from humans. Preformed immune complex (IC) (anti-CD40L antibodies plus soluble CD40 ligand (sCD40L)) was incubated with isolated platelets from four human volunteers; representative data from one volunteer are shown. Traces show the percentage of platelet aggregation over a 6-minute time period from one donor, but is representative of four donors. $\mathbf{b}$ In vitro dense granule release from human platelets. Anti-CD40L antibodies (final concentration $500 \mathrm{nM}$, with/without sCD40L) were incubated with isolated human platelets $(\mathrm{n}=4)$, and ${ }^{14} \mathrm{C}$-radiolabeled serotonin release was measured. Data are plotted as mean percentage serotonin release (average of the means of four donors (SRA)). Error bars are standard deviation for the mean percentage SRA values of the four donors. c In vitro platelet aggregation isolated from Rhesus monkeys. Preformed IC was incubated with isolated platelets from two Rhesus monkeys ( $\mathrm{RH} 1$ and $\mathrm{RH} 2$ ). Traces show the percentage of platelet aggregation over a 6-minute time period from one donor, but is representative of two donors. $\mathbf{d}$ In vitro dense granule release from Rhesus monkey platelets. Preformed IC or antibody solutions (final concentrations $500 \mathrm{nM}$ ) were incubated with isolated platelets from four Rhesus monkeys (black bars) or one healthy human donor (gray bars), and ${ }^{14} \mathrm{C}$-radiolabeled serotonin release was measured. Thrombin receptor agonist peptide (TRAP) (100 $\left.\mu \mathrm{M}\right)$ was included as a positive control. Data are plotted as in $\mathbf{b}$.

determine whether this would translate to a lack of TEs in vivo was undertaken. A head-to-head, 8-week i.v. study to compare the thromboembolic potential of CDP7657 and hu5c8 was conducted in Rhesus monkeys. Hu5c8 at $50 \mathrm{mg} / \mathrm{kg} /$ week i.v. produced widespread pulmonary arterial intravascular thrombosis and/or intimal hyperplasia and associated secondary pathology changes in the lungs (Table 3; Fig. 5a and b). By contrast, CDP7657 and other anti-CD40L antibody constructs lacking a functional Fc region, also administered at $50 \mathrm{mg} / \mathrm{kg} /$ week i.v., were associated with minimal pulmonary changes that were comparable in incidence and severity to those found in 
Table 3 Thromboembolic potential of different anti-CD40L antibody formats in a comparative 8-week study in Rhesus monkeys

\begin{tabular}{|c|c|c|c|c|}
\hline $\begin{array}{l}\text { Dose group } \\
\text { (n) }\end{array}$ & $\begin{array}{l}\text { Total animals } \\
\text { affected (\%) }\end{array}$ & $\begin{array}{l}\text { Total affected } \\
\text { lung sections }\end{array}$ & $\begin{array}{l}\text { Total examined } \\
\text { lung sections }\end{array}$ & $\begin{array}{l}\text { Lung sections } \\
\text { affected (\%) }\end{array}$ \\
\hline $\begin{array}{l}\text { Historic saline } \\
\text { controls }\end{array}$ & $7(50)$ & 10 & 406 & 2.5 \\
\hline \multicolumn{5}{|l|}{$(\mathrm{n}=14)^{\mathrm{a}}$} \\
\hline $\begin{array}{l}\text { Concurrent } \\
\text { saline control }\end{array}$ & $1(25)$ & 1 & 116 & 0.9 \\
\hline \multicolumn{5}{|l|}{$(n=2 / \operatorname{sex})^{a}$} \\
\hline CDP7657 & $3(37.5)$ & 4 & $232(203)^{c}$ & $1.7(2.0)^{c}$ \\
\hline \multicolumn{5}{|l|}{$(n=4 / \operatorname{sex})^{b}$} \\
\hline $\begin{array}{l}\text { Aglycosyl } \\
\text { hu5c8 }\end{array}$ & $3(37.5)$ & 3 & 232 & 1.3 \\
\hline \multicolumn{5}{|l|}{$(n=4 / \operatorname{sex})$} \\
\hline $\mathrm{Hu} 5 \mathrm{c} 8$ & $5(62.5)$ & 41 & 232 & 17.6 \\
\hline$(n=4 /$ sex $)$ & & & & \\
\hline
\end{tabular}

Thrombembolic events were defined as the occurrence of thrombus, an organizing thrombus or intimal hyperplasia. ${ }^{a}$ Historic control group of Rhesus monkeys that had received i.v. saline for up to 6 months; one animal died on day 2, which was unrelated to CDP7657 treatment; ${ }^{C}$ values in parentheses do not include animals removed from the study for humane reasons unrelated to the study saline-treated control animals (Table 3; Fig. 5c and d). In two out of eight hu5c8-treated animals, there was a treatment-related decrease in platelet count (56\% and $42 \%$ on day 37, compared with pre-treatment levels) but this was not observed in any CDP7657-treated animal.

Based on the encouraging in vitro and in vivo data generated with CDP7657, a longer study with higher dose levels of CDP7657 was undertaken. In this study, Rhesus monkeys were dosed weekly for 3 months with CDP7657 at doses up to $200 \mathrm{mg} / \mathrm{kg}$ i.v. No gross or histopathologic findings attributable to CDP7657 were observed in the lungs of treated animals, either at the end of the 3-month treatment period or during a period of up to 6 months following the end of dosing (Table 4). Microscopic thrombi (including mature organizing thrombi) with a similar very low incidence and distribution were observed in both CDP7657-treated and saline-treated control animals. These were considered to be incidental, unrelated to CDP7657, and consistent with background levels observed in historical experiments performed with Rhesus monkeys. Furthermore, no qualitative morphological differences between thrombi were seen between CDP7657treated and saline-treated control groups. At the end of
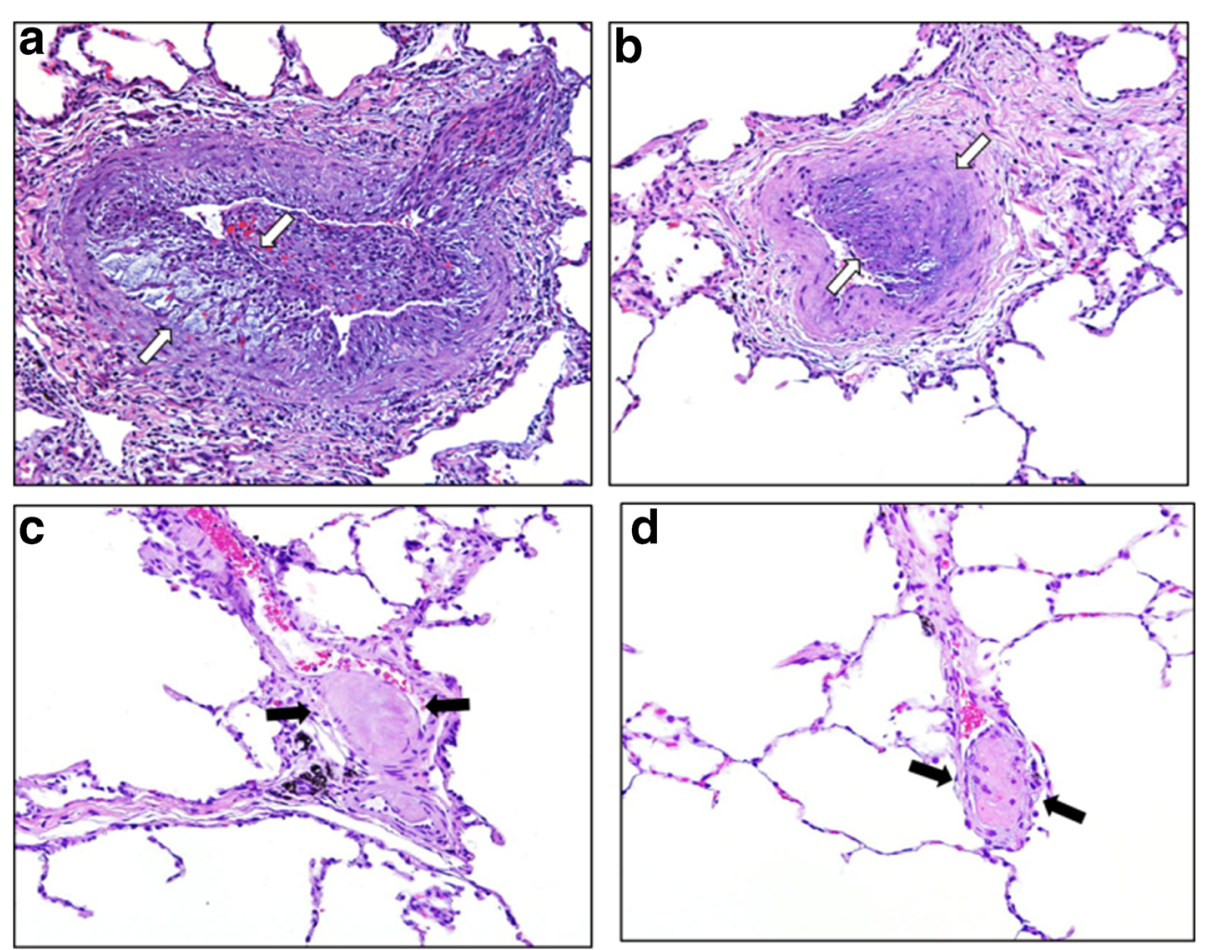

Fig. 5 Histopathology of Rhesus monkey lungs showed no findings attributable to CDP7657. Representative hematoxylin and eosin stained sections of pulmonary arteries/arterioles from Rhesus monkeys that received hu5c8 (a-b), vehicle control (c) or CDP7657 (d). In a and $\mathbf{b}$ arterial thrombus formation is accompanied by marked, irregular thickening of the intimal layer (arrows). Intimal thickening is mediated by in-migration of smooth muscle, deposition of pale basophilic fibrillar extracellular matrix and cleft formation. Vessel lumina contain fibrinocellular debris and entrapped red blood cells. In c and $\mathbf{d}$ fibrin microthrombi adhere to the endothelial lining of thin-walled arterioles (arrows). Under normal conditions, microthrombi are formed and lysed continually within the venous circulatory system. Small pulmonary arterial vessels and capillaries trap thrombi and prevent them from obstructing the vital coronary, cerebral and renal arteries. These observations were isolated, infrequent findings 
Table 4 Thromboembolic potential of CDP7657 in an extended 3-month study in Rhesus monkeys

\begin{tabular}{|c|c|c|c|c|}
\hline Dose group & $\begin{array}{l}\text { Total animals } \\
\text { affected (\%) }\end{array}$ & $\begin{array}{l}\text { Total affected } \\
\text { lung sections }\end{array}$ & $\begin{array}{l}\text { Total examined } \\
\text { lung sections }\end{array}$ & $\begin{array}{l}\text { Lung sections } \\
\text { affected (\%) }\end{array}$ \\
\hline$\overline{\text { Control }^{b}}$ & $10(50)$ & 11 & 581 & 1.9 \\
\hline \multicolumn{5}{|l|}{$(n=10 /$ sex $)$} \\
\hline 20 mg/kg & $6(60)$ & 9 & 290 & 3.1 \\
\hline \multicolumn{5}{|l|}{$(n=5 /$ sex $)$} \\
\hline $50 \mathrm{mg} / \mathrm{kg}$ & $4(40)$ & 4 & 294 & 1.4 \\
\hline \multicolumn{5}{|l|}{$(n=5 /$ sex $)$} \\
\hline $200 \mathrm{mg} / \mathrm{kg}$ & $3(33)$ & 3 & 261 & 1.2 \\
\hline$(n=5 / \operatorname{sex})$ & & & & \\
\hline
\end{tabular}

Thromboembolic events defined as the occurrence of a thrombus or an organizing thrombus (intimal hyperplasia was not observed in this study); ${ }^{a}$ total number of lung sections examined in each group $=29 /$ animal; ${ }^{b}$ treated with i.v. saline

the no-treatment recovery period, microscopic pulmonary thrombi were found in the saline-treated control and CDP7657-treated groups, with a similar low incidence to those found at the end of the dosing period, indicating no association with CDP7567 treatment. Thus, even after prolonged high-dose exposure, there is no evidence that CDP7657 is able to induce TEs in vivo. In addition, no evidence of other toxicity was demonstrated in this study.

The prothrombotic antibody hu5c8 has a fully functional Fc region and is considered to be capable of antigen cross-linking, whereas CDP7657, an agent that is not associated with thrombus formation, does not have an Fc region and is only capable of monovalent binding. Further investigation of the mechanism by which hu $5 \mathrm{c} 8$ promotes thrombus formation showed that treatment with the aglycosyl form of hu5c8 was not associated with thrombus formation (Table 3).

\section{Discussion}

The key role played by the CD40-CD40L axis in regulating T-cell/B-cell interactions makes it a particularly attractive target for the treatment of inflammatory and autoimmune diseases. However, the promise of CD40Lspecific antibodies has been challenged by the association of hu5c8 with TEs $[15,18]$. More recent work has suggested that these unexpected side effects are likely dependent upon the anti-CD40L antibody carrying a functional Fc region [12]. As shown in the pre-clinical studies presented here, CDP7657, a novel high-affinity anti-CD40L PEGylated monovalent Fab' antibody fragment that lacks an Fc region, dose-dependently inhibits the immune response to TT, but does not induce TEs in monkeys.

In this study, CDP7657 and aglycosyl hu5c8, antibody constructs that lack a functional Fc region, strongly inhibited the humoral response to TT, although they had reduced activity compared with hu5c8 (even allowing for plasma exposure), which almost certainly reflects some role for an active Fc in this particular model. Interestingly, aglycosyl hu5c8 has been shown to be effective in the TT immune response model as well as in SLE and experimental autoimmune encephalomyelitis mouse models, but was ineffective in both renal and allograft transplantation models $[23,25]$. This suggests that the contribution of Fceffector function of anti-CD40L antibodies may vary depending on the immune challenge [23]. However, in the current study $60 \mathrm{mg} / \mathrm{kg}$ CDP7657 was still able to abrogate the immune response to the TT antigen to the same degree as hu5c8, despite not possessing an Fc.

As has been highlighted previously, anti-CD40L antibodies are particularly effective in mouse SLE models, showing profound effects on survival as well as proteinuria, autoantibodies and immune cell populations [7]. Interestingly, a more recent study examined disease and mechanistic endpoints in a CD40L ${ }^{-/-}$NZB SLE mouse model [26]. CD40L ${ }^{-/-}$led to abrogated production of autoantibodies, decreases in activated T cells and memory effector cells, lack of germinal center development and reduced plasmablast number. These data corroborate data generated with anti-CD40L antibodies, illustrating that CD40L has an important role in promoting pathogenic autoantibody production and kidney disease in NZB mice, and highlight the potential therapeutic benefit of CD40L blockade as a treatment for autoimmune diseases, particularly those driven by autoantibodies.

In this study, disease remission was seen with a murine anti-CD40L PEGylated monovalent Fab' antibody (MR1 Fab' PEG) in the NZB/W mouse SLE model, using a therapeutic dosing regimen where mice received antibodies only after they had displayed severe proteinuria. Most work that has been published using this model utilized a prophylactic dosing regimen, where treatment was started prior to disease onset. Therapeutic efficacy has been demonstrated previously with the full-length MR1 anti-CD40L antibody [24], but the data presented here suggest that CDP7657, lacking any Fc-function, may have the potential to be an effective therapy in lupus patients with demonstrable disease.

Given the previous history of anti-CD40L antibody evaluation in the clinic, a key requirement for progressing new entities targeting CD40L has been to understand the mechanisms for induction of TE and to design a suitable in vivo model that could demonstrate that the risk of TEs associated with anti-CD40L antibodies has been eliminated. In our studies of isolated human and Rhesus monkey platelets in vitro, activation occurred with hu5c8 IC but not with complexes formed with CDP7657 or other antibody formats lacking an Fc region. Furthermore, although hu $5 \mathrm{c} 8$ was shown to activate human platelets in vitro when it possessed an active Fc moiety, it was without activity in its aglycosyl form in 
these assay systems. Likewise, CDP7657 had no plateletactivating activity in human or Rhesus monkey platelet assays.

These observations are consistent with other studies suggesting a critical role of the IgG Fc regions of antiCD40L antibodies in mediating platelet aggregation [20]. In vitro studies showed that hu5c8/CD40L IC, but not aglycosyl hu5c8 /CD40L IC, activated platelets and that an anti-FcyRIIa monoclonal antibody can block the activation of platelets triggered by intact anti-CD40L/ CD40L IC $[12,20]$. As FcyRIIa is the only IgG Fc receptor detectably expressed on the surface of platelets [27], the involvement of other IgG Fc receptors in these observations is unlikely. More recently, the role of Fc $\gamma$ RIIa in IC-triggered thrombosis was confirmed in transgenic mice [21] where anti-human $\mathrm{CD} 40 \mathrm{~L} / \mathrm{sCD} 40 \mathrm{~L}$ IC triggered a thrombotic response in mice transgenic for human FcyRIIa, but not in wild-type mice. Furthermore, the thrombotic effect observed in FcyRIIa transgenic mice could be triggered by IC comprising soluble mouse CD40L and the anti-mouse CD40L antibody MR1 carrying a human Fc region (hMR1), but not by IC of soluble mouse CD40L and aglycosyl hMR1. Overall, the data suggest a mechanism whereby co-engagement of CD40L and FCR by an intact antibody triggers activation of platelets, possibly following cross-linking events and triggering of signaling; in theory, this could happen in cis, with co-engagement of CD40L and FcR on the same platelet surface, or in trans, mediated by platelet-platelet interactions. In vitro studies published recently with a domain $\mathrm{Ab}$ anti-CD40L antibody, which lacks a functional Fc, similarly concluded that the Fc was essential for platelet activation [28]. These authors also showed that an anti-mouse CD40L domain $\mathrm{Ab}$ was efficacious in a number of mouse models of autoimmune disease.

We have previously published work that suggests that the Rhesus monkey is a relevant and sensitive pre-clinical model for induction of TEs by anti-CD40L IgG $\mathrm{I}_{1}$ antibodies in humans [19]. In the current study, hu5c8 treatment produced extensive pulmonary TEs and pulmonary vasculopathy in Rhesus monkeys. In contrast, no treatmentrelated adverse findings were observed with CDP7657 or aglycosyl hu5c8 and the pulmonary findings observed in CDP7657-treated animals were similar to those observed in saline-treated control animals. No TEs attributable to CDP7657 (using doses up to $200 \mathrm{mg} / \mathrm{kg}$ i.v.) were observed in a 3-month repeat dose safety study, either during the treatment period or after a 6-month treatment-free period, again in contrast to hu5c8. This is the first demonstration that a CD40L antibody lacking the Fc domain, and thus no longer able to activate platelets in vitro, has mitigated the risk of inducing TEs in vivo in Rhesus monkeys.

In all dose groups, including saline control animals, $33-60 \%$ of animals had small, occasional, solitary pulmonary micro-thrombi that were considered to be incidental to injection. It is suspected that these incidental thrombi may originate from the injection site and/or blood sampling venipuncture sites, arising secondary to minor venous trauma [29] and were only observed because of extensive lung tissue sectioning and high examination scrutiny.

Published data suggest that the blockade of CD40L per se would not be expected to cause TE events. Studies in $\mathrm{CD}_{40 \mathrm{~L}^{-/-}}$knockout mice showed protection against thrombosis by genetic deficiency in the CD40L pathway in which accelerated thrombus formation was completely abrogated in arterioles and partially abrogated in venules in $\mathrm{CD}_{40 \mathrm{~L}^{-1-}}$ mice [30]. Exogenous SCD40L reversed this protection. In another study, platelets from CD40L-deficient mice demonstrated reduced shearinduced platelet aggregation [31]. Elevated sCD40L has also been linked to an increased risk of atherothrombosis [32]. It should be stressed that mouse platelets, in contrast to human and non-human primate platelets, do not express FcRs and, therefore, these published studies suggest that blocking CD40L alone would not contribute to an increased incidence of thrombosis and possibly would even suppress thrombosis under certain conditions. Indeed, in our studies, the pulmonary thrombovasculopathy observed with hu5c8 in Rhesus monkeys was dependent on the presence of a functional Fc region, and was not induced by CD40L blockade alone.

\section{Conclusions}

The present studies have demonstrated that anti-CD40L antibodies require a functional $\mathrm{Fc}$ region in order to induce TEs in Rhesus monkeys. CDP7657, a novel high-affinity anti-CD40L PEGylated monovalent Fab' antibody without an Fc region, effectively inhibits CD40L-dependent immune responses. This, in conjunction with the lack of TE and Fcmediated platelet activation, supports the clinical evaluation of this antibody in humans for the treatment of SLE and other autoimmune diseases.

\section{Additional files}

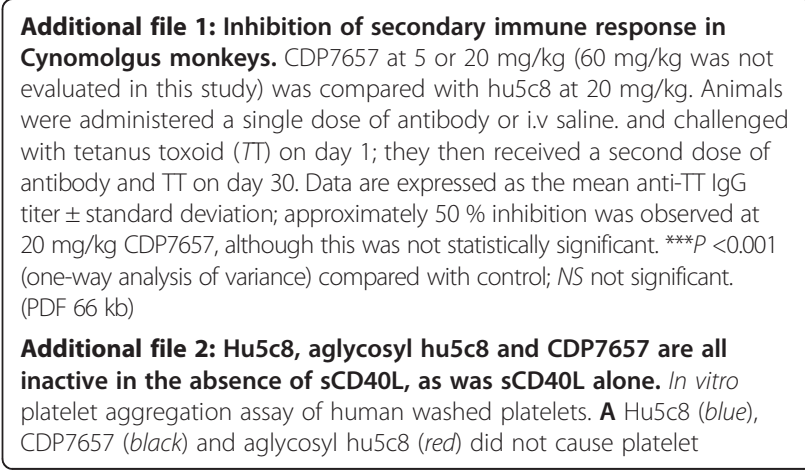


aggregation when introduced to platelets without recombinant human soluble CD40L (rhCD40L). B rhCD40L alone $(10 \mu \mathrm{g} / \mathrm{ml})$ was also inactive. (PDF $180 \mathrm{~kb}$ )

Additional file 3: CDP7657 immune complex (IC) and aglycosyl hu5c8 IC do not induce platelet aggregation in rhesus monkey platelets both in the absence of presence of sub-aggregatory amounts of the platelet agonist (ADP). In vitro aggregation assay of rhesus monkey washed platelets. A CDP7657 IC did not aggregate rhesus monkey washed platelets in the absence (b/ue and red), or presence (black and green) of ADP. B Similarly, aglycosyl hu5c8 IC did not aggregate rhesus monkey washed platelets in the absence (blue and red), or presence (black and green) of ADP. (PDF $171 \mathrm{~kb})$

\section{Abbreviations}

ADP: adenosine diphosphate; ANOVA: analysis of variance; $\mathrm{AUC}_{0-19}$ : area under the concentration-time curve from time 0 until 19 days; CD40L: CD40 ligand; $C_{\text {max }}$ : maximum concentration; ELISA: enzyme-linked immunosorbent assay; FCR: Fc receptor; GLP: Good Laboratory Practice; HRP: horseradish peroxidase; IC: immune complex; ICAM-1: intercellular adhesion molecule-1; i.m.: intramuscular; i.v.: intravenous; $K_{D}$ : equilibrium dissociation constant; kDa: kiloDaltons; NZB/W: New Zealand black/white; PE: phycoerythrin; PEG: polyethylene glycol; rhCD40L: recombinant human soluble CD40L; s.c: subcutaneous; SCD40L: soluble CD40L; SLE: systemic lupus erythematosus; SRA: serotonin release assay; TE: thrombotic events; TRAP: thrombin receptor agonist peptide; TT: tetanus toxoid; TX: treatment.

\section{Competing interests}

Financial competing interests are as follows: AS, CP, SM, AL, TB, NW, SS, OH and AM are employees of UCB Pharma and hold UCB Pharma shares and/or stock options. MR is an ex-employee and paid consultant at UCB Pharma and holds UCB Pharma stock options. IW is an ex-employee and paid consultant at UCB Pharma. RF and DB are former employees of UCB Pharma and have no related competing interests to declare. LB and JF are employees of and hold stock options in Biogen Idec, Inc. EG, FRT, LS and YMH were employees of Biogen Idec, Inc. at the time the study was conducted, and still hold stock options in the company. DH was an employee of Biogen Idec, Inc. at the time of the study. AA, TM and JF have received research support from UCB Pharma. There are no non-financial competing interests to declare for any of the authors.

\section{Authors' contributions}

AS designed the study, performed the experiments, analyzed the data, wrote the manuscript and was involved in the interpretation of the data. LB, IW, $\mathrm{CP}, \mathrm{EG}, \mathrm{JFe}, \mathrm{FRT}, \mathrm{LS}, \mathrm{Y}-\mathrm{MH}, \mathrm{DH}, \mathrm{TM}, \mathrm{JFr}, \mathrm{MR}, \mathrm{SS}, \mathrm{AL}, \mathrm{OH}$ and TB designed the study, analyzed the data and were involved in the interpretation of the data. AA and SM designed the study, performed the experiments, analyzed the data and were involved in the interpretation of the data. DB analyzed the data and was involved in the interpretation of the data. RF and NW designed the study and were involved in the interpretation of the data. AM wrote the manuscript and was involved in the interpretation of the data. All authors critically reviewed the manuscript for important intellectual content and approved the final version.

\section{Acknowledgements}

The study was funded by UCB Pharma. Medical writing and editorial assistance was provided by Lindsay Queen and Lindsay Napier from Darwin Healthcare Communications, and funded by UCB Pharma. The authors thank UCB Pharma employees Julia Steel, Alistair Henry, Helen Neale, Kevin Whale and Marianne Bergin who contributed to the in vitro characterisation of CDP7657, and Joan Lane (Biogen Idec, Inc.) for her review of the in vivo Rhesus monkey data.

\section{Author details}

${ }^{1}$ UCB Pharma, 216 Bath Road, Slough, Berkshire SL1 4EN, UK. ${ }^{2}$ Biogen Idec, Inc., 12 Cambridge Center, Cambridge, MA 02142, USA. ${ }^{3}$ UCB Pharma, Braine, Belgium. ${ }^{4}$ Center for Thrombosis Research, Florida Hospital, Orlando, USA. ${ }^{5}$ Present Address: Ab Biosciences Inc., Allston, MA, USA. ${ }^{6}$ Present Address: Charles River, Wilmington, MA, USA. ${ }^{7}$ Present Address: Cheylard Biosciences, Berkshire, UK. ${ }^{8}$ Present Address: Hammel Ltd, Buckinghamshire, UK.

Received: 7 May 2015 Accepted: 14 August 2015

Published online: 03 September 2015

\section{References}

1. Elgueta R, Benson MJ, de Vries VC, Wasiuk A, Guo Y, Noelle RJ. Molecular mechanism and function of CD40/CD40L engagement in the immune system. Immunol Rev. 2009;229:152-72.

2. Burkly LC. CD40 pathway blockade as an approach to immunotherapy. Adv Exp Med Biol. 2001;489:135-52

3. van Kooten C, Banchereau J. CD40-CD40 ligand. J Leukoc Biol. 2000;67:2-17.

4. Gerritse K, Laman JD, Noelle RJ, Aruffo A, Ledbetter JA, Boersma WJ, et al. CD40-CD40 ligand interactions in experimental allergic encephalomyelitis and multiple sclerosis. Proc Natl Acad Sci U S A. 1996;93:2499-504.

5. Gobburu JV, Tenhoor C, Rogge MC, Frazier DE, Thomas D, Benjamin C, et al. Pharmacokinetics/dynamics of 5c8, a monoclonal antibody to CD154 (CD40 ligand) suppression of an immune response in monkeys. J Pharmacol Exp Ther. 1998;286:925-30.

6. Xu H, Tadaki DK, Elster EA, Burkly LC, Berning JD, Cruzata F, et al. Humanized anti-CD154 antibody therapy for the treatment of allograft rejection in nonhuman primates. Transplantation. 2002;74:940-3.

7. Davidson A, Wang X, Mihara M, Ramanujam M, Huang W, Schiffer L, et al. Co-stimulatory blockade in the treatment of murine systemic lupus erythematosus (SLE). Ann NY Acad Sci. 2003;987:188-98.

8. Toubi E, Shoenfeld Y. The role of CD40-CD154 interactions in autoimmunity and the benefit of disrupting this pathway. Autoimmunity. 2004;37:457-64.

9. Henn V, Slupsky JR, Gräfe M, Anagnostopoulos I, Förster R, Müller-Berghaus $\mathrm{G}$, et al. CD40 ligand on activated platelets triggers an inflammatory reaction of endothelial cells. Nature. 1998;391:591-4.

10. André P, Nannizzi-Alaimo L, Prasad SK, Phillips DR. Platelet-derived CD40L: the switch-hitting player of cardiovascular disease. Circulation. 2002;106:896-9.

11. André P, Prasad KSS, Denis CV, He M, Papalia JM, Hynes RO, et al. CD40L stabilizes arterial thrombi by a beta3 integrin-dependent mechanism. Nat Med. 2002;8:247-52.

12. Langer F, Ingersoll SB, Amirkhosravi A, Meyer T, Siddiqui FA, Ahmad S, et al. The role of CD40 in CD40- $L$ and antibody-mediated platelet activation. Thromb Haemost. 2005;93:1137-46.

13. Elzey BD, Ratliff TL, Sowa JM, Crist SA. Platelet CD40L at the interface of adaptive immunity. Thromb Res. 2011;127:180-3.

14. Semple JW, Freedman J. Platelets and innate immunity. Cell Mol Life Sci. 2010;67:499-511.

15. Boumpas DT, Furie R, Manzi S, Illei GG, Wallace DJ, Balow JE, et al. A short course of BG9588 (anti-CD40 ligand antibody) improves serologic activity and decreases hematuria in patients with proliferative lupus glomerulonephritis. Arthritis Rheum. 2003;48:719-27.

16. Grammer AC, Slota R, Fischer R, Gur H, Girschick H, Yarboro C, et al. Abnormal germinal center reactions in systemic lupus erythematosus demonstrated by blockade of CD154-CD40 interactions. J Clin Invest. 2003;112:1506-20.

17. Huang W, Sinha J, Newman J, Reddy B, Budhai L, Furie R, et al. The effect of anti-CD40 ligand antibody on B cells in human systemic lupus erythematosus. Arthritis Rheum. 2002;46:1554-62.

18. Kawai T, Andrews D, Colvin RB, Sachs DH, Cosimi AB. Thromboembolic complications after treatment with monoclonal antibody against CD40 ligand. Nat Med. 2000;6:114.

19. Wakefield ID, Harari O, Hutto D, Burkly L, Ferrant J, Taylor F, et al. An assessment of the thromboembolic potential of CDP7657, a monovalent Fab' PEG anti-CD40L antibody, in rhesus macaques. Arthritis Rheum. 2010;62:1243.

20. Hsu Y-M, Su L. Platelet aggregation assays using a CD40L-binding agent US patent No. 8409810 B2 (Issued Apr 2, 2013).

21. Robles-Carrillo L, Meyer T, Hatfield M, Desai H, Dávila M, Langer F, et al. Anti-CD40L immune complexes potently activate platelets in vitro and cause thrombosis in FCGR2A transgenic mice. J Immunol. 2010;185:1577-83.

22. Meyer T, Robles-Carrillo L, Robson T, Langer F, Desai H, Davila M, et al. Bevacizumab immune complexes activate platelets and induce thrombosis in FCGR2A transgenic mice. J Thromb Haemost. 2009;7:171-81.

23. Ferrant JL, Benjamin CD, Cutler AH, Kalled SL, Hsu Y-M, Garber EA, et al. The contribution of Fc effector mechanisms in the efficacy of anti-CD154 immunotherapy depends on the nature of the immune challenge. Int Immunol. 2004;16:1583-94.

24. Quezada SA, Eckert M, Adeyi OA, Schned AR, Noelle RJ, Burns CM. Distinct mechanisms of action of anti-CD154 in early versus late treatment of murine lupus nephritis. Arthritis Rheum. 2003;48:2541-54. 
25. Nagelkerken L, Haspels I, van Rijs W, Blauw B, Ferrant JL, Hess DM, et al. FCR interactions do not play a major role in inhibition of experimental autoimmune encephalomyelitis by anti-CD154 monoclonal antibodies J Immunol. 2004;173:993-9.

26. Pau E, Chang NH, Loh C, Lajoie G, Wither JE. Abrogation of pathogenic IgG autoantibody production in CD40L gene-deleted lupus-prone New Zealand Black mice. Clin Immunol. 2011;139:215-27.

27. Anderson CL, Chacko GW, Osborne JM, Brandt JT. The Fc receptor for immunoglobulin G (Fc gamma RII) on human platelets. Semin Thromb Hemost. 1995;21:1-9.

28. Xie JH, Yamniuk AP, Borowski V, Kuhn R, Susulic V, Rex-Rabe S, et al. Engineering of a novel anti-CD40L domain antibody for treatment of autoimmune diseases. J Immunol. 2014:192:4083-92.

29. Lilbert J, Burnett R. Main vascular changes seen in the saline controls of continuous infusion studies in the Cynomolgus monkey over an eight-year period. Toxicol Pathol. 2003;31:273-80

30. Gavins FN, Li G, Russell J, Perretti M, Granger DN. Microvascular thrombosis and CD40/CD40L signaling. J Thromb Haemost. 2011;9:574-81.

31. Crow AR, Leytin V, Starkey AF, Rand ML, Lazarus AH. CD154 (CD40 ligand)deficient mice exhibit prolonged bleeding time and decreased shear-induced platelet aggregates. J Thromb Haemost. 2003;1:850-2.

32. Novo S, Basili S, Tantillo R, Falco A, Davi V, Novo G, et al. Soluble CD40L and cardiovascular risk in asymptomatic low-grade carotid stenosis. Stroke. 2005;36:673-5.

\section{Submit your next manuscript to BioMed Central and take full advantage of:}

- Convenient online submission

- Thorough peer review

- No space constraints or color figure charges

- Immediate publication on acceptance

- Inclusion in PubMed, CAS, Scopus and Google Scholar

- Research which is freely available for redistribution 